\title{
Theoretical Investigation On Standing Wave Thermo-Acoustic Refrigerator: A Review
}

\author{
P. B. Thorat, P. S. Pandure, A. A. Patil \\ (Department Of Mechanical Engineering, M. E. S. College Of Engineering, S. P. Pune University, India)
}

\begin{abstract}
Thermoacoustic Refrigerator is a device which uses the high intensity acoustic waves in a pressurized gas tube to pass heat from one place to other to produce cooling effect. Thermoacoustic technology is proposed in this study as an alternative to current issues with conventional refrigerator because of there is no any environmental hazards refrigerants during its working. The system does not use refrigerants like Chlorofluorocarbons (CFCs), Hydro-chlorofluorocarbons (HCFCs) which are toxic chemicals and it results in the depletion of ozone layer but uses inert gases like Helium, Argon etc. or air at latm instead. It does not require any moving parts except loudspeaker connected to a signal generator that produces the acoustics. Thermoacoustic refrigeration classifies in two types based on standing wave which is compact system and travelling wave which is generally a large system. The concept of thermoacoustic refrigeration system is explain, various investigations into thermoacoustic refrigeration system, different techniques to improve COP, different stacks which is the heart of this system and resonator tube designs to improve heat transfer rate. The literature review gives a complete picture on the history and the exiting situation of thermoacoustic refrigeration system.
\end{abstract}

Keywords : Thermoacoustic, Refrigerator, standing wave, COP, stack

\section{Introduction}

Modern refrigeration technologies were introduced in the 19th century, their use has significantly increased. Now it is impossible to living without refrigeration and air conditioning system. Conventional refrigerator use the ozone depleting chemical namely Freon gas, which is being compressed to absorb the heat. This compressed Freon liberates chlorine molecules which causes depletion of ozone layer present in the atmosphere. The most important requirement for the working fluid is its volumetric cooling capacity and boiling point. In 19th century natural refrigerants like carbon dioxide (Co2), ammonia (NH3), and Sulphur dioxide (So2) are used but it requires a relative high operating pressure to achieve useful boiling point. Pure form of the gases is not used as working fluid. There are alternative technologies to provide cooling lieu of vapour compression devices. One that can provide refrigeration without any refrigerant is the thermoacoustic refrigeration. The common thermoacostic refrigerator is sown in Fig.1 [1,3].

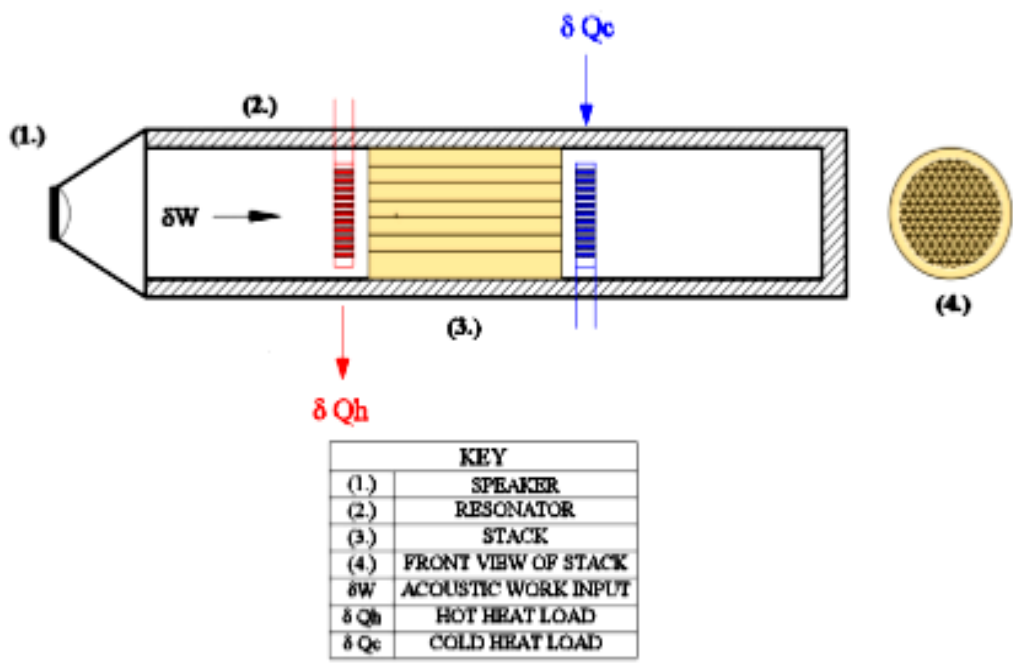

Fig.1 Sound wave thermoacoustic Refrigerator [5] 
There are two types of TARs, first is standing wave thermoacoustic refrigerator which uses fixed number of oscillation and does not change for long interval of time. This is similar as the guitar string fixed at two ends. And second is travelling wave thermoacoustic refrigerator it uses the sound wave that travel across the TAR. Each type of system has specific advantages in certain situations. TARs are still less efficient than vapor compression refrigerators [7].Thermoacoustic refrigeration operates based on the fundamental thermodynamic heat pump where work is necessary in this case acoustical work to transfer heat from low temperature body to a high temperature body. The loudspeaker produces an acoustic standing wave at the fundamental frequency which interacts with a porous medium known as stack and long hollow tube is called as resonator which store acoustic energy and obtain proper phasing of pressure and velocity. The stack is made of many narrow passages separated by thin plates [3].In 2004 we could get close to the commercial thermoacoustic refrigerator. Ben and Jerry's ice cream had a thermoacoustic refrigerator fitted to their one of the ice-cream freezer [7].

\section{Optimization And Performance Analysis}

The performance of the refrigerator is defined as the ratio of the transferred heat at the cold heat exchanger and acoustic power use to transfer the heat [3]. Tijani et al [3] explained in detail the designing criteria for thermoacoustic refrigerator in order to achieve an optimal system, use the linear thermoacoustic theory to describe design criteria. Dimensionless independent variables were used to decrease the number of parameters and to simplify equations. He result reported give guidance on the identification of highest performance based on the coefficient of performance (COP). Tijani et al achieved a low temperature of $-65^{\circ} \mathrm{C}$ is one of the lowest reported temperatures of the system. Stack length, porosity, stack position and acoustic frequency are all variables which influence efficiency or the performance of system. Tijani et al studied outcome of plate spacing and geometry of plate in the stack on the performance of the device. There are many geometries of stack like a. spiral, b. parallel plate, c. honeycomb pores, d. corning ceclor etc. shown in Fig.2.

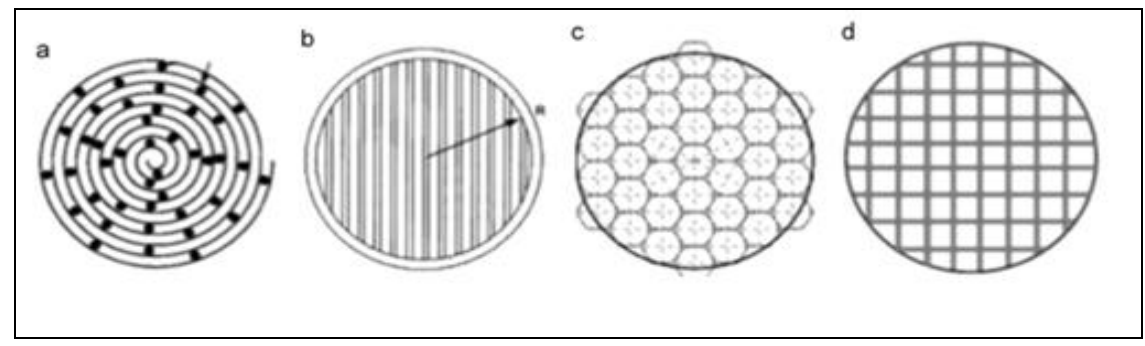

Fig.2 Types of Stacks

The analysed stack material made by low thermal conductivity material that is photographic film or Mylar material is selected. The selection of an average pressure of 10bar, and the helium as working gas, determines the thermal and viscous penetration depth.A.C. Alcock et al investigated that the use of frequency and coupled geometry parameters as non-independent variables and investigation of possible interdependent relationship. Result increases the feasibility of this technology being introduced as thermoacoustic refrigerator. In Fig. 3, the resonant frequency was determined for each stack position. The graph shows that resonant frequencies for each stack position are different. Fig. 4 shows the relation between the temp difference across the stack and stack position. It indicates that there is maximum expected temperature difference when stack located closer to the pressure antinode (closed end).

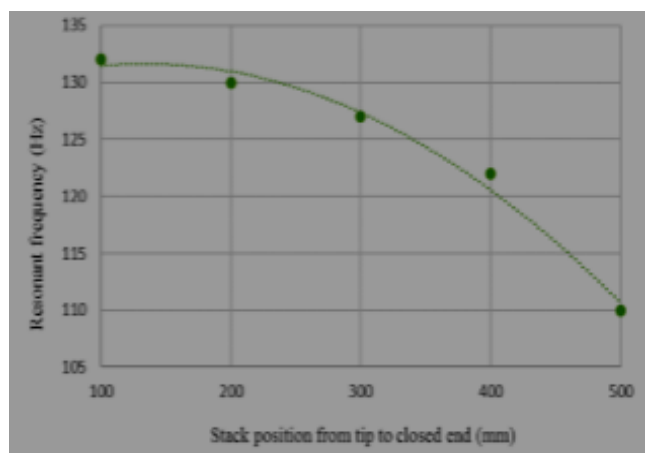

Fig.3: Size 1 stack-resonant frequency vs stack position

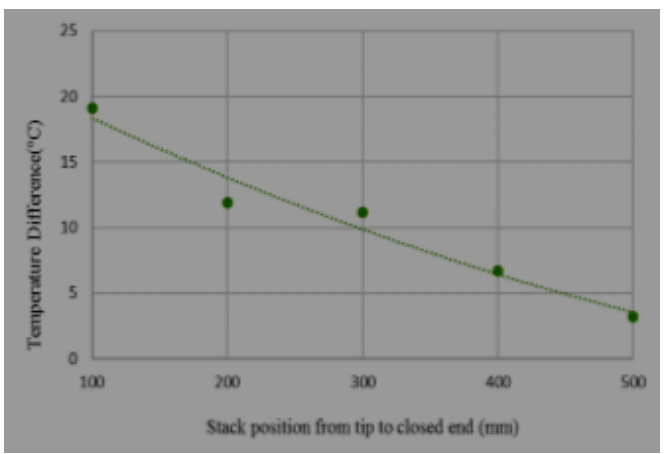

Fig. 4: Size 1 stack-resonant frequency vs stack 
Bheemsha et al. [4] explained the optimization and design of a thermo acoustic refrigeration system with the help of general linear theory of thermo acoustics, as the basis for the design taking its simplified assumptions into consideration. Optimization was carried out using MATLAB. Hriharan et al. [5] investigated that the COP thermoacoustic refrigeration system calculated by means of temperature variation along stack, by two dissimilar spacing i.e. $0.4 \mathrm{~mm}$ and $0.8 \mathrm{~mm}$. The temperature difference was higher in case of mylar sheet than photographic film with $0.4 \mathrm{~mm}$ spacing. As the spacing increases, the temperature difference between the stack decreases. From the experiment it is observed that at the beginning temperature difference increases with time increasing and after some interval it stabilized with time. This is occurred due to smooth convection between working fluid and plate (Fig 5).In case of hot end temperature, when hot end of stack is higher then there is increase in temperature difference between the stack for different material which results into the better performance of the system.

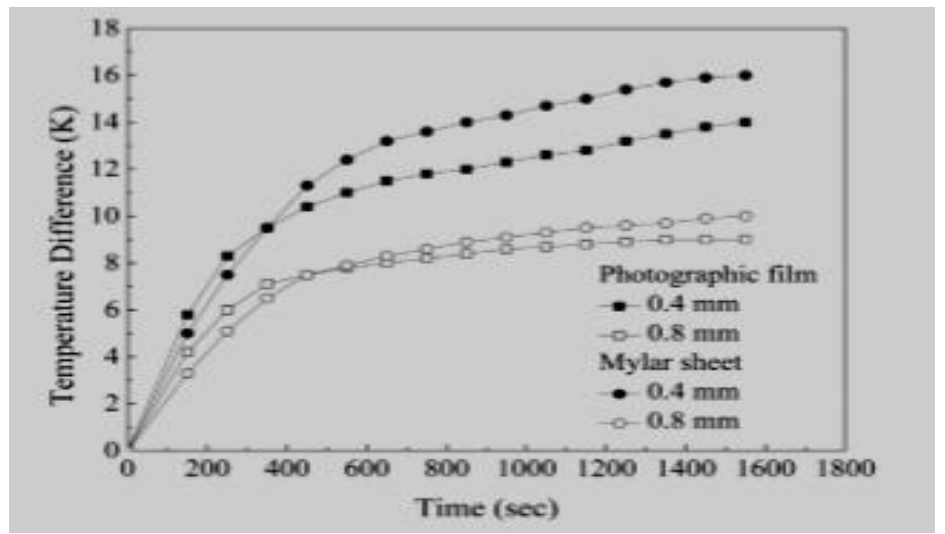

Fig.5 Temperature difference vs time for different stack with different spacing

Jithin George [6] investigated the affect of resonator tube length. The length of resonator increases it will increases the hot end temperature. For improving the performance aluminium plug is replaces with plastic plug.. Giulio Allesina analysed for small diameter resonator and rolled stack, the temperature difference (Delta $\mathrm{T}_{\max }$ ) is approximately $24^{\circ} \mathrm{C}$. While using the parallel plate stack with same fundamental parameters the result shows that temperature difference is less than about $25 \%$ of rolled stack. This different result is occurred due to the difference of effective area of the two stacks.N. A. Zolpakar et al [7] analysed the heat transfer between gas and the wall occurs within a thermal boundary layer where pure conduction takes place. He investigated that heat transfer area should be large for the cooling in large amount. So the stack is generally made of many solid walls that are separated by 2 and 4 thermal penetration depth. If the wall of stack are too close or too far apart, effective heat transfer between the stack wall and gas packet cannot occur effectively. Hence the pin array stack is selected as perfect stack which has large amount of thermal boundary layer.N. A. Zoplakar [8] analysed that a standing wave thermoacoustic stack having low thermal conductivity and high heat capacity. So the RVC is preferred for latest stack material. But the mylar is the best stack material to date because of low thermal conductivity which help to prevent diffusion of heat through the stack. Also the stack length and stack position are variables investigated. These variables are inter-dependent where the increase in stack length increases the stack centre position, measured from the acoustic driver. Researchers have discovered that stack centre position is between lambda/ 8 and lambda/20 and these positions are dependent on stack length.The above mentioned literature is based on optimization methods of thermoacoustic refrigerator which include linear theoretical method, analytical and software method. The linear theoretical method is concentrated only on resonator tube length, and plate spacing. Whereas the numerical and analytical methods are used for optimization of temperature across the resonator tube, stack length, frequency of working fluid, stack position, plate spacing etc, with the help of CATIA, MATLAB software. All this methods are used for optimization of thermoacoustic refrigeration system. Optimization is nothing but the improvement of systems towards better performance; ultimately all this methods are used to improve the COP of thermoacoustic refrigeration systems.

\section{Conclusion}

- The desired separation gap between solid walls should be between 2 and 4 thermal boundary layer. The stack with large spacing decreases the heat transfer rate and viscous effect increases due to stack with small spacing.

- $\quad$ Pin array with high boundary layer is selected as perfect stack geometry.

- Air is used as working fluid because it is easily available but the sound velocity is high in helium gas.

6th National Conference RDME 2017, 17th-18th March 2017. 
- For designing the stack mylar is the best material.

- When the design of stack is as small as possible, the thermal losses are minimized and when design is large the power output is maximized.

- The low temperature of the system reported is $-65^{\circ} \mathrm{C}$ up to date.

- Thermoacoustic refrigerators are likely to be lightweight, compact, robust and does not contain any harmful refrigerants.

\section{References}

[1]. Tijani M.E.H., Zeegers J.C.H., and De Waele A.T.A.M, Design of thermoacoustic refrigerators, Cryogenics, 42 (1),2002, $49-57$.

[2]. N.M. Hariharan, P. Sivashanmugam, S. Kasthurirengan, Experimental investigation of a thermoacoustic refrigerator driven by a standing wave twin thermoacoustic prime mover, International Journal of Refrigeration, 36( 8),2013,2420-2425.

[3]. Tijani M.E.H., Zeegers J.C.H., and De Waele A.T.A.M., Construction and performance of a thermoacoustic refrigerator, Cryogenics, 42,2002,59-66.

[4]. Bheemsha, R. Nayak. B, Pundarika.G, Design of the Resonator Tube and Buffer Volume for Thermo Acoustic Refrigerator, International journal of Advanced Scientific and Technical Research, 2, 2011, 215-220.

[5]. A.C. Alcock, L.K. Tartibu, T.C. Jen, Experimental investigation of ceramic substrates in standing wave thermoacoustic refrigerator, Procedia manufacturing, 7,2016,79-85.

[6]. Jithin George, Loud speaker driven thermoacoustic refrigeration, International Journal of Scientific and Engineering Research, 7(4),2016,2229-5518.

[7]. N.Atiqah Zolpkar, Normah Mohd-Ghazali,Mawahib Hassan El-Fawal, Performance analysis of the standing wave thermoacoustic refrigerator:A review, Renewable and Sustainable Energy Reviews, 54,2016,626-634.

[8]. G. Allesina, An experimental analysis of stand-alone standing wave thermoacoustic refrigerator, International Gernal of Energy and Environmental Engineering, 1,2014,5-4. 